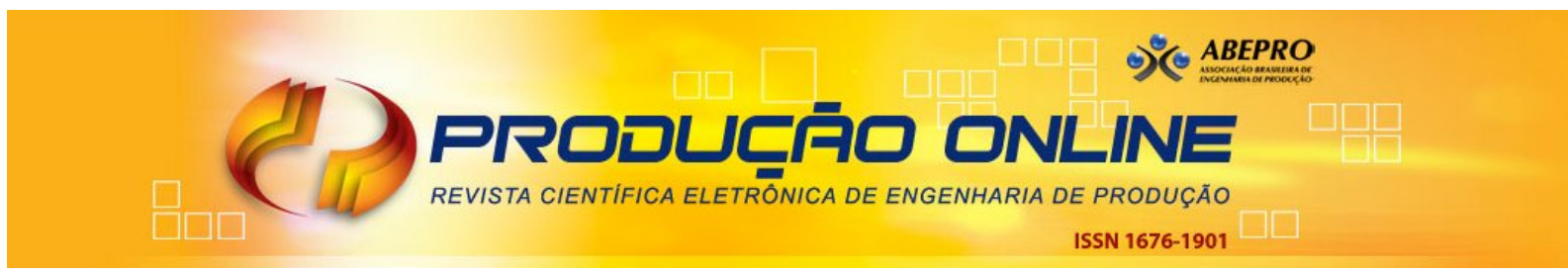

\title{
O PAPEL DO CAPITAL RELACIONAL (CR) NA GESTÃO VERDE DA CADEIA DE SUPRIMENTOS (GVCS)
}

\section{THE ROLE OF RELATIONAL CAPITAL (RC) IN GREEN SUPPLY CHAIN MANAGEMENT (GSCM)}

\author{
Cláudia Viviane Viegas* E-mail: claudiavv@unisinos.br \\ Caroline Rodrigues Vaz ${ }^{\star \star}$ E-mail: caroline-vaz@hotmail.com \\ Paulo Maurício Selig** E-mail: pauloselig@gmail.com \\ Gregório Varvarkis** E-mail: grego@egc.ufsc.br \\ *Universidade do Vale do Rio dos Sinos (UNISINOS), São Leopoldo (RS), Brasil \\ ${ }^{\star *}$ Universidade Federal de Santa Catarina, (UFSC), Florianópolis (SC), Brasil
}

\begin{abstract}
Resumo: Gestão Verde da Cadeia de Suprimentos (GVCS) é um tema emergente na gestão de operações voltado à adoção de práticas intra e interorganizacionais para estimular a sustentabilidade em cadeias produtivas. Capital Relacional (CR), também um tema emergente, tem papel relevante para redimensionar as visões baseadas unicamente em custos de transação e recursos para uma visão baseada em relações que envolvam governança de informações, confiança e longevidade nas relações, buscando resultados positivos para todos os integrantes da cadeia. Este artigo revisa e critica a literatura seminal de ativos intangíveis para resgatar atributos de CR e relacioná-los ao desenvolvimento da GVCS. Utilizando-se de método exploratório e descritivo, são identificados estudos recentes sobre CR e GVCS quanto aos seus descritores - formas como o CR é entendido e utilizado na GVCS; foco - finalidade principal do uso do CR; e direcionalidade - se os desenvolvimentos do CR na GVCS são direcionados do produtor a seus fornecedores e clientes, ou se estes últimos também direcionam resultados do CR. Conclui-se que, em geral, há unidirecionalidade de aplicação desses esforços: iniciativas de melhoria partem do produtor para o desenvolvimento do fornecedor ou do cliente, mas pouco se exploram os processos pelos quais práticas ambientais de fornecedores e clientes influenciam o desempenho na GVCS.
\end{abstract}

Palavras-chave: Gestão Verde da Cadeia de Suprimentos. Capital relacional. Ativos intangíveis.

\begin{abstract}
Green Supply Chain Management (GSCM) is an emergent issue in operations management. It is addressed to intra and inter-organizational practices adoption in order to promote sustainability in supply chains. Relational Capital (RC), also an emergent issue, plays a relevant role to resize views based solely in transactions costs and resources to a relationship view leaned on governance for information, trust and longevity in relationships, looking for positive results for all the chain's participants. This article reviews and criticizes seminal literature on intangible assets in order to rescue RC features and link them to GSCM development. Through exploratory and descriptive methods, recent studies on RC and GSCM are identified with respect their descriptors - ways in which $\mathrm{RC}$ is understood and employed in GSCM; focus - goals and main use of RC; and directionality - if developments of RC in GSCM are addressed from producers to their suppliers and respective clients, or if suppliers and clients also address results of RC. It is concluded that, usually, there is unidirectionality in the application of such efforts: improvement initiatives go from the producer to the supplier or client development, but processes through which environmental practices of suppliers and clients influence the performance of GSCM are poor explored.
\end{abstract}

Keywords: Green Supply Chain Management. Relational Capital. Intangible Assets.

Revista Produção Online, Florianópolis, SC, v.15, n. 3, p. 980-998, jul./set. 2015. 


\section{INTRODUÇÃO}

A Gestão Verde da Cadeia de Suprimentos Verde (GVCS) é um tema emergente na gestão de operações voltado à adoção de práticas intra e interorganizacionais visando a criar, manter e incrementar a combinação de desempenhos ecológico, econômico e social que estão no âmbito de influência das organizações participantes da cadeia produtiva. Embora os conceitos de GVCS e Gestão Sustentável da Cadeia de Suprimentos (GSCS) tenham ganhado delineamentos diferenciados entre si, especialmente a partir da recente revisão sistemática de ambos realizada por Ahy e Searcy (2013), é difícil deixar de considerar a significativa coincidência de abrangência quanto às classificações "verde" e "sustentável" no âmbito de estudo da cadeia produtiva, principalmente porque os próprios autores expõem conceitos que indicam claras sobreposições entre ambos, mesmo afirmando que GSCS é mais abrangente que GVCS. Ashby et al. (2012), que também revisaram estudos relacionando a forma como a sustentabilidade é abordada na cadeia produtiva, observam que o aspecto sustentável ainda está polarizado nas iniciativas ambientais e que a dimensão social é subprepresentada, apesar de sua relevância. Estas constatações iniciais reforçam a escolha de direcionamento do presente estudo: por um lado, é muito difícil isolar o aspecto ambiental quando se trata da sustentabilidade da cadeia produtiva, embora ele seja ainda dominante; por outro, há uma declarada falta de investigação dos aspectos sociais, que são, por natureza, relacionais, ou seja, compreendem as formas como sujeitos representam e operam com conceitos e ferramentas da cadeia que se pretende "verde" ou "sustentável".

Estudos recentes dão conta da importância de compreender e sistematizar as relações interorganizacionais para a criação de valor na GCVS, o que pode ser traduzido como o estudo e a explicitação do capital relacional (CR) necessário a fornecedores e clientes em qualquer estágio da cadeia produtiva. Contudo, o CR é dificilmente destacável dos demais tipos de ativos intangíveis - como os capitais humano e estrutural. Isto dificulta a tarefa de compreensão de como o CR influencia os fluxos de trocas entre integrantes da cadeia produtiva. Faltam pesquisas para explicar como ocorrem as relações entre fornecedores e clientes, e poucas são as análises que conectam aspectos ambientais e sociais (VIEGAS et al., 2014). Revista Produção Online, Florianópolis, SC, v.15, n. 3, p. 980-998, jul./set. 2015. 
Rajaguru e Matanda (2013) identificam um vácuo investigativo para explicar os fatores de integração de sistemas interorganizacionais que dizem respeito às capacidades dinâmicas dos agentes. Assim, mais dificuldades somam-se para desafiar o avanço nos estudos da GVCS: a literatura escassa sobre como o fornecedor é envolvido nas demandas do comprador (DOU et al., 2014) e a explicitação dos fatores que facilitam a formação e o aprimoramento do CR interfirmas (BLONSKA et al., 2013).

O objetivo deste estudo exploratório é identificar conceitos e descritores de CR na literatura de ativos intangíveis, mesmo desafiando concepções tradicionais; e explicitar o significado e o direcionamento dessas relações na GVCS. O pressuposto teórico do trabalho está na incorporação de uma visão ulterior de relacionamento com respeito à explicitada pela da Teoria dos Custos de Transação (TCT), que considera como passíveis de contabilização custos de riscos inerentes à negociação interfirmas focados principalmente na possibilidade de quebras de contratos. Está também num avanço sobre a Visão Baseada em Recursos (VBR), na qual o centro de análise é o desenvolvimento e uso de competências, de natureza relacional ou não. Diferentemente dos fatores assumidos pela TCT e pela VBR, resgatam-se aspectos da Visão Relacional (VR), considerada uma extensão da VBR e segundo a qual relações próximas e colaborativas são a chave para o estabelecimento da vantagem competitiva entre clientes e fornecedores (RIBBKIN e GRIMM, 2014).

O trabalho está assim dividido: na seção 2, são apresentados o método e os procedimentos da pesquisa; na seção 3, são apresentados os resultados, em duas etapas: na subseção 3.1, são explicitados atributos e conceitos de CR, analisandose tanto a literatura seminal de capital intelectual quanto os significados de relacional em GVCS; na subseção 3.2 são explicitados os descritores de CR na literatura da GVCS (3.2.1), o foco da relação em cada estudo (3.2.2) e a direcionalidade (3.2.3), ou seja, se o desenvolvimento das relações se dá apenas a partir do cliente para o fornecedor, se há bidirecionaldade na relação, ou se o desenvolvimento é autodirecionado (da organização para ela mesma). Na seção 4 são apresentadas as conclusões, limitações e recomendações para pesquisas futuras. 


\section{MÉTODO E PROCEDIMENTOS}

Este estudo é de natureza exploratória (GIL, 2002) devido à ausência de pesquisas teóricas relacionando CR e GVCS. É descritivo porque se propõe a levantar e explicitar as características do objeto em estudo - CR na GVCS (SANTOS, 2002). Para seu desenvolvimento, foi inicialmente realizada uma pesquisa sobre os principais conceitos de CR presentes na literatura clássica de ativos intangíveis e na literatura de GVCS. Também foi realizada uma busca avançada na base de dados Science Direct (SD), para o período 2004-2014, utilizando as expressões "green supply chain management" e "relational capital", inicialmente com a primeira expressão para "todos os campos" e a segunda para "título, resumo e palavras-chave", e depois no sentido inverso, ou seja, com a primeira expressão para "título, resumo e palavras-chave" e a segunda para "todos os campos".

Os procedimentos de trabalho incluíram a sistematização de atributos e conceitos de CR, com a crítica e releitura desse constructo no contexto da GVCS. Incluíram também a compilação e análise do que se convencionou classificar como: (i) descritores ou atributos mais importantes do CR; (ii) foco dos mesmos considerando as ferramentas e procedimentos "verdes" adotados em cadeias produtivas dos estudos selecionados; e (iii) direcionalidade do CR na cadeia "verde", ou seja, se as relações de "esverdeamento" na cadeia produtiva são dirigidas do produtor para o fornecedor e seus clientes, se o movimento inverso é o que ocorre, ou se há bidirecionalidade.

\section{RESULTADOS}

Na primeira parte dos resultados são destacados atributos e conceitos de CR selecionados da literatura clássica - sendo desafiada a classificação estabelecida por alguns autores, visando à composição de um quadro conceitual o mais completo e conforme as ideias de fluxo, troca e dinâmica entre atores da cadeia produtiva.

$\mathrm{Na}$ segunda parte, são apresentados os resultados das duas buscas na base de dados Science Direct, nas quais obteve-se um total de 13 estudos: dois considerados pertinentes em conteúdo, de um total de quatro, na primeira busca; e Revista Produção Online, Florianópolis, SC, v.15, n. 3, p. 980-998, jul./set. 2015. 
11 considerados também pertinentes em conteúdo, de um total de 12, na segunda busca. Nesta segunda seção também são analisados os resultados conforme as classificações observadas nos estudos quanto aos "constructos" de CR "foco" das ações ou ferramentas "verdes" empregadas, e "direcionalidade" das iniciativas "verdes" no relacionamento entre produtores e seus fornecedores e clientes.

\subsection{Atributos e conceitos de CR}

As principais definições de CR provêm da literatura de Capital Intelectual ( $\mathrm{Cl}$ ) - muitas vezes chamado de "ativos intangíveis" ou mesmo "ativos invisíveis", pois envolve atributos abstratos, difíceis de definir, medir e gerenciar (BONTIS, 1998). Antes de definir $\mathrm{CR}$, é relevante examinar o que a literatura seminal - desenvolvida especialmente a partir da década de 90 - apresenta como capital intelectual e como insere o $\mathrm{CR}$ no $\mathrm{Cl}$. A classificação mais comum para $\mathrm{Cl}$ refere-se ao mesmo como um misto de capital humano (habilidades, conhecimento tácito), capital estrutural (dependente do conhecimento das pessoas e das estruturas organizacionais) e CR (referente às relações envolvendo clientes, fornecedores, colaboradores e investidores (ROOS e ROOS,1997; SVEIBY, 1997; LYNN, 1998).

Para efeitos de mensuração, o capital humano é desdobrado em competências (habilidades e saber fazer), atitudes (comportamento, motivação, liderança), agilidade intelectual (BONTIS et al., 1999; PABLOS, 2004) e até mesmo capacidade de inovação (COSER, 2012).

Capital estrutural é compreendido como estrutura organizacional tangível, em forma física, ou intangível, composta por habilidades, experiências e conhecimentos institucionalizados - explicitados por meio da tecnologia da informação ( $\mathrm{TI}$ ), patentes, manuais, rotinas, fluxogramas, protocolos e procedimentos da organização, processos, cultura e valores empresariais (EDVINSSON e MALONE, 1998). Um dos aspectos-chave do capital estrutural é o capital de processos, que deve atender expectativas de clientes por meio do cumprimento de metas de produção e gestão de qualidade adequadas. Este capital é avaliado a partir da satisfação do cliente (ESCAFFRE, 2002). Riopel et al. (2011) observam que os sistemas de informação, 
como parte do capital estrutural, facilitam a comunicação e a colaboração entre indivíduos no processo de logística.

Já o $\mathrm{CR}$, como parte do $\mathrm{Cl}$, é entendido como o conjunto de relações que envolve a organização, seu entorno e os decorrentes feedbacks (STEWART, 1998), sendo também descrito como o valor gerado pelas relações da empresa com seus clientes, como, por exemplo, soluções de problemas pós-venda (EDVINSSON e SULLIVAN, 1996). É ainda apontado como todos os ativos que derivam de relações com o mercado (BROOKING, 1996) e as inovações consequentes dessas relações (GUBIANI, 2011).

É possível observar que as descrições de capitais humano e estrutural envolvem também elementos relacionais, que somente se efetivam por meio de contatos e trocas entre agentes interna e externamente às organizações. Portanto, competências, atitudes - enquadradas como capital humano - e habilidades, experiências, processos, sistemas de informação - caracterizados como capital estrutural - são também atributos de capital relacional. Da mesma forma, capacidade de inovação - tratada pela literatura de $\mathrm{Cl}$ como um atributo de capital humano e estrutural, ao mesmo tempo, é também relacional porque depende fundamentalmente das relações entre agentes.

Esta crítica da classificação do $\mathrm{Cl}$ para reposicionamento pragmático do CR é fundamental no sentido de dirimir o excesso de rigidez classificatória e estabelecer clareza a fim de que se possam melhor compreender as funções dos atributos de relação na GVCS. A este respeito, Nardone et al. (2010) alertam quanto ao risco de falácia em se classificar todos os intangíveis como "capital social" ou mesmo "intelectual" sem considerar os atributos de laços entre os agentes da cadeia produtiva ou seja, os movimentos práticos que dão base e sentido à realização das relações, como atitude, lealdade, preferência e outros aspectos emocionais e cognitivos que impulsionam os relacionamentos: "[Q]ualquer análise empírica concluirá que capital social causa cooperação entre agentes e melhora a eficiência de mercados" (SABATINI, 2009, apud NARDONE et al., 2010, p. 65). Da mesma forma, Sydler et al. (2014) reconhecem que é difícil separar o CR dos demais tipos de capital porque a interligação dos atributos que os compõem é que dá diferencial único a cada organização. Assim, o CR é entendido como não exatamente como 
relações, mas como o valor delas com partes externas, fornecedores, distribuidores, lobby das organizações, parceiros com os quais a empresa mantém relações de lealdade, preferências e reconhecimento da marca (SYDLER et al., 2014).

Os aspectos vistos prioritariamente como subjetivos - reciprocidade, confiança, solidariedade, cooperação - recebem como agregadores a ideia de governança, sendo inclusive possível cogitar no índice de capital relacional como a razão entre o número de relações existentes e o número máximo de relações possíveis entre agentes de organizações em interação (NARDONE et al., 2010). A visão mais recente de $\mathrm{CR}$ em cadeias produtivas constata a coexistência dos paradigmas transacional - relações predominantemente adversas entre fornecedores e clientes - e relacional - relações mutuamente benéficas entre as partes (LEE et al., 2010). Pablos-Herdero et al. (2012, p. 133), neste contexto, definem capital relacional como "a habilidade das pessoas de trabalhar em grupo conforme regras e valores compartilhados", articulando habilidades e conhecimentos adquiridos em processos de comunicação para gerar vantagens competitivas.

Contudo, não se podem ignorar fatores que levam também ao relacionamento não colaborativo, como assinala Bresnen (2007). Este autor adverte sobre paradoxos em relações de parcerias ou alianças que surgem devido a diferenças culturais entre as partes envolvidas, bem como a relações assimétricas de poder; à perseguição de patamares excelentes de desempenho, praticamente impossíveis de se atingir, capazes de abrir fontes de insatisfação e ressentimentos nas relações; e ao estrangulamento possível dos processos de criatividade e aprendizagem nas relações interfirmas devido à excessiva padronização de processos. Embora tais aspectos não possam ser ignorados, no presente trabalho são levados em consideração os atributos relacionais que geram entregas positivas para as partes envolvidas quanto ao desempenho ambiental da cadeia produtiva em que se inserem.

O Quadro 1 apresenta um sumário dos atributos de $\mathrm{Cl}$ em sua classificação mais usual (clássica) e a releitura crítica de alguns deles como CR. 
Quadro 1 - Visões clássicas e críticas do CR

\begin{tabular}{|c|c|c|c|}
\hline $\begin{array}{l}\text { CAPITAL } \\
\text { INTELECTUAL }\end{array}$ & $\begin{array}{l}\text { ATRIBUTOS } \\
\text { "CLÁSSICOS" }\end{array}$ & \multirow{4}{*}{ 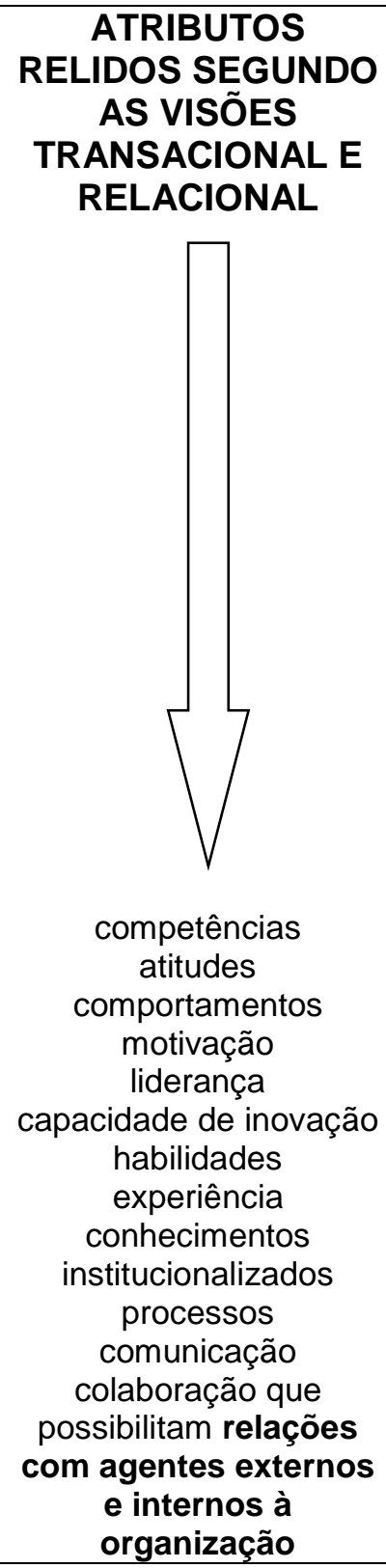 } & \multirow{4}{*}{ 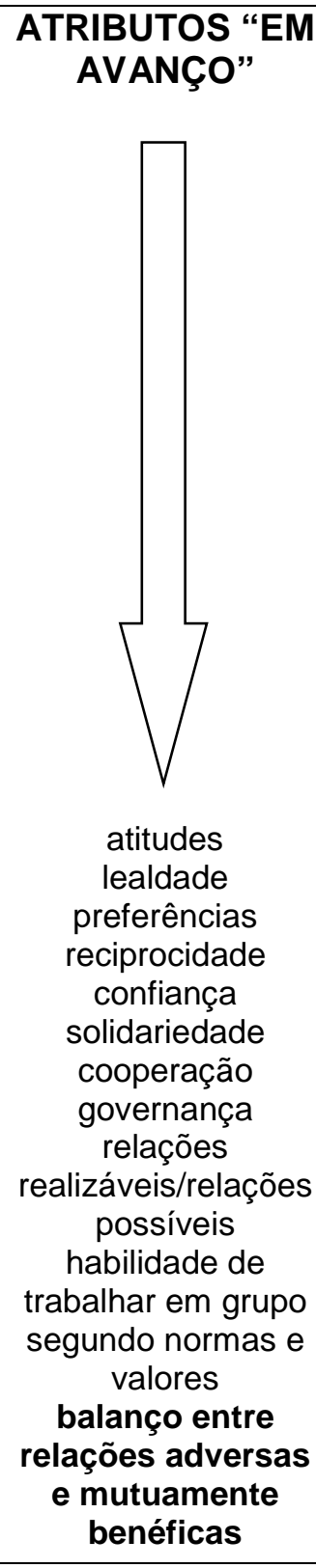 } \\
\hline Humano & $\begin{array}{c}\text { competências } \\
\text { atitudes } \\
\text { comportamentos } \\
\text { motivação } \\
\text { liderança } \\
\text { agilidade intelectual } \\
\text { capacidade de inovação }\end{array}$ & & \\
\hline Estrutural & $\begin{array}{c}\text { habilidades } \\
\text { experiência } \\
\text { conhecimentos } \\
\text { institucionalizados } \\
\text { TI } \\
\text { processos } \\
\text { comunicação } \\
\text { colaboração } \\
\text { capacidade de inovação }\end{array}$ & & \\
\hline Relacional & $\begin{array}{l}\text { relações com agentes } \\
\text { externos e internos à } \\
\text { organização }\end{array}$ & & \\
\hline
\end{tabular}

Fonte: Elaborado pelos autores

\subsection{Descritores, foco e direcionalidade do CR na GVCS}

GVCS é compreendida como um conjunto de estratégias e ações que incluem cooperação com fornecedores e consumidores para ecodesign, produção mais limpa $(\mathrm{P}+\mathrm{L})$, redução do uso de energia, compra "verde", gestão ambiental interna e recuperação de investimentos via ações de logística reversa (LR) (ZHU et al., 2013). Chan et al. (2012) consideram GVCS como três conjuntos de práticas: compra 
verde, colaboração com fornecedores para ações de gestão ambiental e recuperação do investimento (por revenda, reaproveitamento ou reciclagem).

A colaboração com fornecedores está em praticamente todos os aspectos técnicos da GVCS, caracterizando sua predominância relacional. A recuperação do investimento é mais conhecida como LR - uma parte significativa da GVCS por meio da qual são gerenciados fluxos de materiais do seu ponto de consumo até o seu ponto de origem, envolvendo etapas de coleta, classificação, tratamento, sistema de informação, expedição e coordenação de materiais (RIOPEL et al., 2012).

Analisando-se 13 estudos recentes e selecionados sobre GVCS e capital relacional, conforme exposto no método deste trabalho, são doravante destacados descritores, foco e direcionalidade desses estudos com relação aos papeis dos principais atores - produtores, seus fornecedores e clientes. A esquematização desta análise está no Quadro 2.

\subsubsection{Descritores}

Consideram-se descritores os atributos que caracterizam o $\mathrm{CR}$, sendo o mais comum nos estudos a colaboração entre as partes da cadeia produtiva "verde" (AZEVEDO et al., 2013; CANIËLS et al., 2013; CHAN et al., 2012; DOU et al., 2014; VACHON e KLASSEN, 2008; ZHU et al., 2013). A colaboração é entendida como encorajamento para a adoção de comportamentos ambientais resilientes (AZEVEDO et al., 2013), prontidão dos fornecedores perante requerimentos significantes dos consumidores (CANIËLS et al., 2013), cooperação para satisfazer variáveis de design ecológico, Produção Mais Limpa $(P+L)$, redução do consumo de energia, e variáveis de recuperação de investimentos - criando mecanismos de diálogo que permitam a negociação constante entre as partes (CHAN et al., 2012). Dou et al. (2014) entendem como colaboração a propensão dos fornecedores em se envolver em programas de desenvolvimento para a GVCS, sendo relevante o porte do fornecedor para um engajamento mais efetivo. Vachon e Klassen (2008, p. 295) veem a colaboração interorganizacional como "planejamento ambiental compartilhado e trabalho conjunto para reduzir a poluição ou outros impactos ambientais", tendo igual peso e responsabilidade para fornecedores e produtores 
para a tomada de decisões. Contudo, muito da relação colaborativa provém de pressões institucionais e econômicas (ZHU et al., 2012).

Os descritores de CR na GVCS aparecem na literatura recente como um misto entre aspectos já conhecidos nos estudos de gestão ambiental - transferência de tecnologia (BAI e SARKIS, 2010), desenvolvimento de fornecedores (FU et al., 2012), comprometimento da alta administração (OLUGU et al., 2011), integração entre parceiros da cadeia (VACHON e KLASSEN, 2006) - e aspectos de caráter mais subjetivo - confiança e busca de longevidade nas relações entre as partes (HOEJMOSE et al., 2012; BLONSKA et al., 2013), confiança e socialização (MEEHAN e BRYDE, 2014), e ética na cadeia de suprimentos (OLUGU et al., 2011). Há outros descritores intermediários entre os já conhecidos e os subjetivos: aprendizagem (CHAN et al., 2012), criação de conhecimento relacional (MEEHAN e BRYDE, 2014) e capacidade de governança de suprimento (BLONSKA et al., 2013) - forma de compartilhamento de informações e decisões entre integrantes da cadeia para melhor atingir metas.

Entre os estudos analisados, dois merecem especial atenção pelo seu conteúdo detalhado das conexões entre CR e GVCS: o de Blonska et al. (2013) e o de Meehan e Bryde (2014). Segundo Bonska et al. (2013), o avanço mais significativo nos estudos relacionais de GVCS está na transição da visão puramente transacional, referente aos custos de gestão de contratos e riscos, para uma visão mais abrangente, caracterizada como colaborativa ou relacional. Estes autores argumentam que "o desenvolvimento do fornecedor encapsula dois blocos construtivos de capital relacional: conhecimento compartilhado e investimentos em transações específicas" (BLONSKA et al., 2013, p. 1295). Isto significa que o CR é uma instância posterior à do desenvolvimento do fornecedor na cadeia de suprimentos. Contudo, a pesquisa existente até então não mostra bem como ocorre o desenvolvimento do fornecedor. Meehan e Bryde (2014) compartilham a ideia de que a análise do CR na GVCS deve ser considerada além dos limites imediatos das relações entre compradores e vendedores na cadeia produtiva, mas tomam como referência de análise somente a perspectiva dos produtores. Em geral, segundo a crítica de Blonska et al. (2013), há uma tendência das análises a deterem-se no estudo dos benefícios da eficiência operacional e não na criação de valor mútuo em 
longo prazo, o que implicaria a inclusão de atributos como lealdade, que leva a investimentos na longevidade das relações.

\subsubsection{Foco}

A análise do foco significa identificar, nos estudos selecionados, para qual finalidade é dirigido o esforço de CR na GVCS. O mais comum é a melhoria de processos e/ou o desenvolvimento conjunto, entre fornecedor e produtor, de soluções de sustentabilidade (BLONSKA et al., 2013; DOU et al., 2014; HOEJMOSE et al., 2012). São citados especificamente por Vachon e Klassen (2006) e Vachon e Klassen (2008) os focos em melhoria de qualidade, flexibilidade, entregas e custos decorrentes de iniciativas ambientais, como $\mathrm{P}+\mathrm{L}$ - esta mencionada também por Azevedo et al. (2013) e Chan et al. (2012).

Redução de consumo de materiais (AZEVEDO et al., 2012), energia (CHAN et al., 2012), compra "verde" (OLUGU et al., 2011), ecodesign (BAI e SARKIS, 2010) e Logística Reversa (LR) (CHAN et al., 2012; ZHU et al., 2013) são focos bem mais específicos, assim como atendimento à legislação (FU et al., 2012). Aumento do nível de informação e de troca de informações entre fornecedor e produtor para a melhoria do desempenho ambiental é um foco relevante de governança citado por Meehan e Bryde (2014). Bai e Sarkis (2010) enfatizam a análise do ciclo de vida do produto, e Caniëls et al. (2013) não explicitam um foco de análise. É importante destacar que há sempre a tentativa de conciliação entre o desempenho ecológico e os ganhos em custos.

\subsubsection{Direcionalidade}

Direcionalidade implica verificar em que direção o CR está produzindo efeitos: se apenas na perspectiva unidirecional (do produtor demandando esforços do fornecedor para economias "verdes" e/ou do cliente, para recuperação de investimentos, como, por exemplo, na LR), ou na perspectiva bidirecional (com o fornecedor sendo protagonista e/ou coautor dos efeitos do CR). Nos estudos analisados, cinco referem-se a descrições de ações unidirecionais, do produtor para 
o fornecedor e/ou deste para o consumidor (AZEVEDO et al., 2013; BAI e SARKIS, 2010; CHAN et al., 2012; DOU et al., 2014; FU et al., 2012). A análise predominante do sentido em que ocorre o desdobramento do CR na GVCS mostra o que já é constatado por Blonska et al. (2013): que, em geral, o desenvolvimento do fornecedor ou do cliente para a sustentabilidade é visto apenas sob a ótica do produtor, e não o oposto.

Como há diferenças entre o empenho de esforços para desenvolver capacidades dos fornecedores e o empenho para desenvolver seus comportamentos, é mais usual que o CR seja empregado primeiramente nas capacidades operacionais e de processo. As capacidades relativas a comportamentos, atitudes e atributos de relacionamento pessoal, como confiança, lealdade e busca de longevidade nas relações são vistas como mais trabalhosas e difíceis de monitorar. Esta dificuldade é bem detalhada por Bresnen (2007), para quem as relações de parceria com ganhos mútuos são difíceis mais de manter do que de alcançar devido à sua intensa dependência de contextos sociais, econômicos e institucionais alheios às iniciativas dos parceiros da cadeia.

Em geral, a pesquisa não considera o CR como um construto mediador, especialmente do lado do fornecedor. O desenvolvimento de fornecedores se realiza muito mais sob a perspectiva técnica - de qualidade, entrega, gestão de custos - do que de governança - com compartilhamento de informações para benefícios mútuos (BLONSKA et al., 2013). Chan et al. (2012) corroboram que, até há pouco tempo, a pesquisa pouco explorava como a orientação ambiental dos agentes influenciava as práticas ambientais corporativas e o desempenho da empresa - o que está, gradativamente, sendo modificado pela maior inserção do CR.

Foram identificados três estudos em que se verifica a existência de unidirecionalidade de ações (do produtor para o fornecedor ou consumidor), mas com análise bidirecional das relações na GVCS: os de Blonska et al. (2013), Hoejmose et al. (2012), Caniëls et al. (2013). Isto significa que tais pesquisas, mesmo focadas no protagonismo do produtor, analisam os efeitos do CR sobre outras partes (fornecedores e/ou clientes). Outros três estudos foram identificados como totalmente bidirecionais em desenvolvimento e análise: os de Olugu et al. (2011), Vachon e Klassen (2006) e Vachon e Klassen (2008). Eles tanto descrevem 
quanto analisam os desenvolvimentos relacionais, para frente e pra trás, na cadeia produtiva "verde". Os outros dois estudos - de Meehan e Bryde (2014) e de Zhu et al. (2013) - são autodirecionais, ou seja, analisam iniciativas adotadas por produtores para o desenvolvimento de atributos de melhoria ambiental, com apoio dos fornecedores (ZHU et al., 2013), mas voltadas aos aspectos internos da organização protagonista do desenvolvimento.

Quadro 2 - Descritores, foco e direcionalidade do CR na GVCS

\begin{tabular}{|c|c|c|}
\hline DESCRITORES & FOCO & DIRECIONALIDADE \\
\hline \multicolumn{3}{|c|}{ Azevedo et al. (2013) } \\
\hline $\begin{array}{l}\text { colaboração } \\
\text { capacitação } \\
\text { monitoramento }\end{array}$ & $\begin{array}{l}\text { ecodesign, logística reversa, } \\
\text { redução de consumo energético } \\
P+L\end{array}$ & $\begin{array}{l}\text { produtor para fornecedor } \\
\text { (capacitação) e produtor para } \\
\text { cliente (monitoramento), } \\
\text { unidirecional }\end{array}$ \\
\hline \multicolumn{3}{|c|}{ Bai e Sarkis (2010) } \\
\hline $\begin{array}{l}\text { comunicação } \\
\text { aconselhamento } \\
\text { treinamento } \\
\text { transferência de } \\
\text { tecnologia }\end{array}$ & $\begin{array}{l}\text { avaliação de tecnologias } \\
\text { "verdes" } \\
\text { análise do ciclo de vida de } \\
\text { produtos } \\
\text { ecodesign }\end{array}$ & $\begin{array}{l}\text { produtor para fornecedor, } \\
\text { unidirecional }\end{array}$ \\
\hline \multicolumn{3}{|c|}{ Blonska et al. (2013) } \\
\hline $\begin{array}{l}\text { governança de } \\
\text { suprimentos } \\
\text { confiança } \\
\text { longevidade das relações } \\
\text { controle e dependência }\end{array}$ & $\begin{array}{l}\text { desenvolvimento conjunto de } \\
\text { produtos } \\
\text { melhoria de processos }\end{array}$ & $\begin{array}{l}\text { investimento do produtor no } \\
\text { fornecedor, mas análise de } \\
\text { resultados sob o ponto de } \\
\text { vista do fornecedor } \\
\text { busca de benefícios mútuos - } \\
\text { bidirecionalidade }\end{array}$ \\
\hline \multicolumn{3}{|c|}{ Caniëls et al. (2013) } \\
\hline $\begin{array}{l}\text { prontidão dos } \\
\text { fornecedores } \\
\text { normas e exigências dos } \\
\text { consumidores } \\
\text { tamanho das empresas } \\
\text { em relação }\end{array}$ & $\begin{array}{l}\text { não especificado - todas as } \\
\text { etapas da cadeia produtiva }\end{array}$ & $\begin{array}{l}\text { investimento do produtor no } \\
\text { desenvolvimento do } \\
\text { fornecedor } \\
\text { participação do fornecedor nas } \\
\text { iniciativas do produtor - } \\
\text { bidirecionalidade }\end{array}$ \\
\hline \multicolumn{3}{|c|}{ Chan et al. (2012) } \\
\hline $\begin{array}{l}\text { colaboração com } \\
\text { fornecedores e clientes } \\
\text { orientação ambiental } \\
\text { estratégica } \\
\text { aprendizagem } \\
\text { relações } \\
\text { interinstitucionais } \\
\text { consideração de } \\
\text { diferentes pontos de vista } \\
\text { na negociação } \\
\text { saber ouvir } \\
\text { evitar oportunismo }\end{array}$ & $\begin{array}{l}\text { compra "verde" } \\
\text { P+L } \\
\text { logística reversa } \\
\text { redução do consumo de energia } \\
\text { redução de materiais de } \\
\text { embalagens }\end{array}$ & $\begin{array}{l}\text { orientação do produtor para o } \\
\text { fornecedor e do produtor para } \\
\text { o consumidor - unidirecional } \\
\text { em ambos os casos }\end{array}$ \\
\hline
\end{tabular}

Revista Produção Online, Florianópolis, SC, v.15, n. 3, p. 980-998, jul./set. 2015. 
Quadro 2 - Descritores, foco e direcionalidade do CR na GVCS

\begin{tabular}{|c|c|c|}
\hline DESCRITORES & FOCO & DIRECIONALIDADE \\
\hline $\begin{array}{l}\text { propensão do fornecedor } \\
\text { a se engajar } \\
\text { relações/contratos de } \\
\text { longo prazo } \\
\text { treinamento e tomada de } \\
\text { decisão estratégicas }\end{array}$ & $\begin{array}{l}\text { melhoria de processos } \\
\text { produtivos (mais "verdes") }\end{array}$ & $\begin{array}{l}\text { transferência de } \\
\text { conhecimento, recursos, } \\
\text { investimento em práticas e } \\
\text { comunicação do produtor para } \\
\text { o fornecedor - unidirecional }\end{array}$ \\
\hline \multicolumn{3}{|c|}{ Fu et al. (2012) } \\
\hline $\begin{array}{l}\text { desenvolvimento de } \\
\text { fornecedores "verdes" }\end{array}$ & $\begin{array}{l}\text { redução ou eliminação de } \\
\text { materiais no processo } \\
\text { atendimento à legislação } \\
\text { ambiental } \\
\text { desenvolvimento de processos } \\
\text { ecológicos }\end{array}$ & $\begin{array}{l}\text { transferência de } \\
\text { conhecimento, recursos, } \\
\text { investimento em práticas e } \\
\text { comunicação do produtor para } \\
\text { o fornecedor - unidirecional }\end{array}$ \\
\hline \multicolumn{3}{|c|}{ Hoejmose et al. (2012) } \\
\hline $\begin{array}{l}\text { estabelecimento de } \\
\text { mecanismos de confiança } \\
\text { e lealdade entre parceiros }\end{array}$ & $\begin{array}{l}\text { melhoria de processos } \\
\text { redução de custos e riscos de } \\
\text { desenvolvimento de produtos }\end{array}$ & $\begin{array}{l}\text { estabelecimento de códigos de } \\
\text { conduta, normas, } \\
\text { monitoramento e auditoria do } \\
\text { produtor para o fornecedor - } \\
\text { orientação unidirecional e } \\
\text { análise bidirecional }\end{array}$ \\
\hline \multicolumn{3}{|c|}{ Meehan e Bryde (2014) } \\
\hline $\begin{array}{l}\text { socialização } \\
\text { confiabilidade } \\
\text { normas compartilhadas } \\
\text { existência de agentes } \\
\text { intermediários } \\
\text { compartilhamento e } \\
\text { criação de conhecimento } \\
\text { relacional }\end{array}$ & $\begin{array}{l}\text { melhorias operacionais } \\
\text { aumento do nível de informação } \\
\text { sobre sustentabilidade para } \\
\text { fornecimento }\end{array}$ & $\begin{array}{l}\text { auto-análise dos atores quanto } \\
\text { a aspectos operacionais, } \\
\text { cognitivos e sociais de } \\
\text { compras sustentáveis - } \\
\text { autodirecionalidade }\end{array}$ \\
\hline \multicolumn{3}{|c|}{ Olugu et al. (2011) } \\
\hline $\begin{array}{l}\text { comprometimento da alta } \\
\text { administração com } \\
\text { gestão ambiental } \\
\text { cadeia de suprimentos } \\
\text { "ética" }\end{array}$ & $\begin{array}{l}\text { compra "verde" } \\
\text { produção "verde" } \\
\text { gestão e distribuição ecológica } \\
\text { de materiais }\end{array}$ & $\begin{array}{l}\text { análise da cadeia de } \\
\text { suprimentos par a frente - } \\
\text { compradores - e para trás - } \\
\text { fornecedores - } \\
\text { bidirecionalidade }\end{array}$ \\
\hline \multicolumn{3}{|c|}{ Vachon e Klassen (2006) } \\
\hline $\begin{array}{l}\text { integração entre } \\
\text { organizações parceiras }\end{array}$ & $\begin{array}{l}\text { implementação de } \mathrm{P}+\mathrm{L} \\
\text { ecodesign } \\
\text { compra verde } \\
\text { melhoria de custos, qualidade, } \\
\text { entregas, flexibilidade e redução } \\
\text { de danos ambientais }\end{array}$ & $\begin{array}{l}\text { desenvolvimento conjunto de } \\
\text { medidas para incrementar } \\
\text { processos e prevenir danos } \\
\text { ambientais por parte de } \\
\text { produtores e fornecedores - } \\
\text { ação e análise bidirecionais }\end{array}$ \\
\hline \multicolumn{3}{|c|}{ Vachon e Klassen (2008) } \\
\hline $\begin{array}{l}\text { colaboração } \\
\text { planejamento ambiental } \\
\text { compartilhado }\end{array}$ & $\begin{array}{l}\text { melhoria de custos, qualidade, } \\
\text { entregas, flexibilidade e redução } \\
\text { de danos ambientais }\end{array}$ & $\begin{array}{l}\text { ações voltadas do produtor ao } \\
\text { fornecedor e vice-versa, e do } \\
\text { produtor ao consumidor, e } \\
\text { vice-versa - bidrecionalidade } \\
\text { em ambos os casos }\end{array}$ \\
\hline \multicolumn{3}{|c|}{ Zhu et al. (2013) } \\
\hline $\begin{array}{l}\text { pressões institucionais } \\
\text { ações colaborativas }\end{array}$ & $\begin{array}{l}\text { ecodesign } \\
\text { gestão ambiental interna } \\
\text { compra "verde" logística reversa }\end{array}$ & $\begin{array}{l}\text { Iniciativas de GCSV adotadas } \\
\text { pelo produtor com apoio de } \\
\text { fornecedores - } \\
\text { autodirecionalidade }\end{array}$ \\
\hline
\end{tabular}

Fonte: elaborado pelos autores 


\section{CONCLUSÕES, LIMITAÇÕES E SUGESTÕES PARA ESTUDOS FUTUROS}

Os temas CR e GVCS têm desenvolvimento recente na literatura, e a inserção de ativos relacionais na gestão ambiental de cadeias produtivas carece de estudos que descrevam e expliquem melhor a forma como atributos de CR influenciam as iniciativas de sustentabilidade no âmbito interorganizacional. O conceito de $\mathrm{CR}$ é disperso na literatura de $\mathrm{Cl}$, o que dificulta sua delimitação e identificação de atributos.

A presente pesquisa propôs, inicialmente, uma contribuição para a superação de barreiras conceituais em torno da ideia de CR. Fez isto ao identificar os atributos relacionais em outros tipos de $\mathrm{Cl}$ - humano e estrutural - consagrados na literatura de ativos intangíveis.

Na segunda parte, a pesquisa identificou 13 estudos sobre CR e GVCS e analisou seus descritores, ou seja, como tais estudos podem ser caracterizados de acordo com os requisitos de CR. Concluiu que a colaboração é o descritor mais utilizado, embora estejam emergindo outros mais subjetivos, como confiança, lealdade, benevolência e longevidade das relações.

Foram também analisados o foco e a direcionalidade dos estudos GVCS sob a ótica do CR. Quanto ao foco, verificou-se que melhoria de processos e desenvolvimento de ações conjuntas entre produtores e fornecedores são os mais explorados nos estudos. Já em relação à direcionalidade, a análise mostrou que os produtores são os principais mentores, direcionadores e beneficiários dos esforços de uso do CR na GVCS. Em geral, há unidirecionalidade nesses esforços, isto é, as iniciativas de melhoria partem do produtor para o desenvolvimento do fornecedor ou do cliente, mas pouco se exploram os processos pelos quais práticas ambientais de fornecedores e clientes influenciam o desempenho na GVCS.

Esta pesquisa apresenta limitações de escopo. A primeira delas é considerar o conceito de GVCS não excludente de todos os aspectos da sustentabilidade, ou seja, considerar a "cadeia verde" como "sustentável", incluindo aspectos econômicos e sociais além dos relativos a melhorias ecológicas. Esta consideração vai aparentemente de encontro à coleção conceitual de Ahi e Searcy (2013), que elencaram conceitos para "gestão verde" (GVCS) e para "gestão sustentável" 
(GSCS) da cadeia de suprimentos após revisarem 180 estudos nesta temática. Contudo, uma análise amiúde dos conceitos expressos por esses autores mostra significativos pontos de sobreposição em termos de conteúdo, o que justificou a presente escolha pelo termo GVCS.

Uma segunda limitação da pesquisa está na seleção de aspectos construtivos de relações interfirmas para o estabelecimento de iniciativas "verdes" ou de "sustentabilidade" com ganhos para a cadeia, sem analisar paradoxos que decorrem da busca de parcerias em sentido amplo - como as relações assimétricas de poder, as diferenças culturais e o risco do excesso de padronização apontados por Bresnen (2007). Dada a delimitação de CR como um conjunto de ativos intangíveis para além das visões baseada em recursos e de custos de transação, a agenda positiva de CR é a que se optou por seguir nesta análise, o que não impede uma problematização futura quanto a possíveis efeitos negativos, para partes da cadeia produtiva, da implementação de iniciativas "verdes" ou "sustentáveis". Finalmente, a utilização apenas de uma base de dados para a busca de estudos analisados está justificada no caráter exploratório do estudo, dado que não foram identificados trabalhos semelhantes relativos a questões como foco e direcionalidade de esforços de gestão verde ou sustentável associados a CR no âmbito das cadeias de suprimentos. Recomenda-se, para pesquisas subsequentes, o refinamento dos descritores de CR, considerando estudos críticos sobre as relações interfirmas, e a ampliação da amostragem de artigos a serem analisados quanto à relação CR e GVCS.

\section{REFERÊNCIAS}

AHI, P.; SEARCY, C. A comparative literature analysis of definitions for green and sustainable supply chain management. Journal of Cleaner Production 52: 329-341, 2013. DOI: http://dx.doi.org/10.1016/j.jclepro.2013.02.018.

ASHBY, A.; LEAT, M.; HUDSON-SMITH, M. Making connections: a review of supply chain management and sustainability literature. Supply Chain Management: An International Journal. 17/5 (2012) 497-516. DOI: http://dx.doi.org/10.1108/13598541211258573.

AZEVEDO, S.G.; GOVINDAN, K.; CARVALHO, H.; CRUZ-MACHADO, V. Ecosilient Index to assess the greenness and resilience of the upstream automotive supply chain. Journal of Cleaner Production 56, p.131-146, 2013. DOI: http://dx.doi.org/10.1016/j.jclepro.2012.04.011. 
BAI, C.; SARKIS, J. Green supplier development: analytical evaluation using rough set theory. Journal of Cleaner Production 18, p.1200-10, 2010. DOI:

http://dx.doi.org/10.1016/.j.jclepro.2010.01.016.

BLOSNKA, A.; STOREY, C.; ROZEMEIJER, F.; WETZELS, M.; RUYTER, K. Decomposing the effect of supplier development on relationship benefits: The role of relational capital. Industrial Marketing Management, v. 42, Issue 8, p.1295-1306, 2013. DOI:

http://dx.doi.org/10.1016/j.indmarman.2013.06.007.

BONTIS, N. Intellectual capital: an exploratory study that develops measures and models. Management Decision, V. 36, N. 2, p. 63-76, 1998. DOI: http://dx.doi.org/10.1108/00251749810204142.

BONTIS, N.; DRAGONETTI, N.C.; JACOBSEN, K.; ROSS, G. The knowledge toolbox: A review of the tools available to measure and manage intangible resources. European Management Journal, V. 17, N. 4, p. 391-402, 1999. DOI: http://dx.doi.org/10.1016/S02632373(99)00019-5.

BRESNEN, M. Deconstructing partnering in project-based organisation: Seven pillars, seven paradoxes and seven deadly sins. International Journal of Project Management 25, p. 365-374, 2007. DOI: http://dx.doi.org/10.1016/j.ijproman.2007.01.007.

BROOKING, A. Intellectual Capital: core assets for the third millennium enterprise. Thomson Business Press, London, United Kingdom, 1996.

CANIËLS, M.C.J.;GEHRSITZ, M. H.; SEMEIJN, J. Participation of suppliers in greening supply chains: An empirical analysis of German automotive supplier. Journal of Purchasing \& Supply Management 19, p. 134-143, 2013. DOI: http://dx.doi.org/10.1016/i.pursup.2013.02.005.

CHAN, R.Y.K.; HE, H.; CHAN, H.K.; WANG, W.Y.C. 2012. Environmental orientation and corporate performance: The mediation mechanism of green supply chain management and moderating effect of competitive intensity. Industrial Marketing Management 41: 621-630. DOI: http://dx.doi.org/10.1016/j.indmarman.2012.04.009.

COSER, A. Modelo para análise da influência do capital intelectual sobre a performance dos projetos de software. Tese (Doutorado). Programa de Pós-graduação em Engenharia e Gestão do Conhecimento, Universidade Federal de Santa Catarina, Florianópolis, 220p., 2012.

DOU, Y.; ZHU, Q.; SARKIS, J. Evaluating green supplier development programs with a greyanalytical network process-based technology. European Journal of Operational Research 233, pp. 420-431, 2014.DOI: http://dx.doi.org/10.1016/j.ejor.2013.03.004.

EDVINSSON, L.; SULLIVAN, P. Developing a Model for Managing Intellectual Capital. European Management Journal, N. 4, p.356-364, 1996. DOI: http://dx.doi.org/10.1016/0263-2373(96)00022-9.

EDVINSSON, L.; MALONE, M. S. Capital intelectual. Descobrindo o valor real de sua empresa pela identificação de seus valores internos. São Paulo: Makron Books, 1998. 
ESCAFFRE, L. Contribution a l'analyse des determinants de l'offre d'information sur le capital intellectual. Tese (Doutorado em Ciência de Gestão), Université Paris IX Dauphine, Paris, 262 p, 2002.

FU, X.; ZHU, Q.; SARKIS, J. Evaluating green supplier development programs at telecommunications systems providers. Int. J. Production Economics 140, p. 357-367, 2012. DOI: http://dx.doi.org/10.1016/j.ijpe.2011.08.030.

GIL, A.C. Como elaborar projetos de pesquisa. São Paulo: Atlas 4. ed., 2002.

GUBIANI, J.S. Modelo para diagnosticar a influência do capital intelectual no potencial de inovação nas universidades. Tese (Doutorado). Programa de Pós-graduação em Engenharia e Gestão do Conhecimento. Universidade Federal de Santa Catarina, Florianópolis, 197p., 2011.

HOEJMOSE, S.; BRAMMER, S.; MILLINGTON, A. "Green" supply chain management: The role of trust and top management in B2B and B2C markets. Industrial Marketing Management, V 41, Issue 4, p. 609-620, 2012. DOI: http://dx.doi.org/10.1016/j.indmarman.2012.04.008.

LEE, W.; LIN, T.M.Y., LEE, W.; LEE, J.R. Relational and transactional factors as hybrid criteria for buyer project selection (BPS): An exploratory study from industrial suppliers. Industrial Marketing Management 39, p. 605-615, 2010. DOI: http://dx.doi.org/10.1016/j.indmarman.2009.05.003 .

LYNN, B.E. Intellectual capital: unearthing hidden value by managing intellectual assets. Ivey Business Journal, Toronto, Jan./Feb, p. 48-52, 2000.

MEEHAN, J.; BRYDE, D. Procuring sustainability in social housing: The role of social capital. Journal of Purchasing \& Supply Management, artigo em publicação, 8p. DOI: http://dx.doi.org/10.1016/j.pursup.2014.01.002 ., 2014.

NARDONE, G.; SISTO, R.; LOPOLITO, A. Social Capital in the LEADER Initiative: a methodological approach. Journal of Rural Studies 26, p. 63-72, 2010. DOI: http://dx.doi.org/10.1016/j.jrurstud.2009.09.001.

PABLOS, P.O. Measuring and reporting structural capital: Lessons from European learning firms. Journal of Intellectual Capital, V 5, N. 4, p. 629-647, 2004. http://dx.doi.org/10.1108/14691930410567059.

PABLOS-HERDERO, C.; BOTELLA, J.L.M.; LOS SANTOS, I.S. The effectiveness of open innovation practices: an application to efficient replenishment. Procedia Technology 5, p.133-140, 2012. DOI: http://dx.doi.org/10.1016/j.protcy.2012.09.015 .

OLUGU, E. U.; WONG, K.Y.; SHAHAROUN, A.M. Development of key performance measures for the automobile green supply chain. Resources, Conservation and Recycling, V 55, Issue 6, p. 567-579, 2011. DOI: http://dx.doi.org/10.1016/j.resconrec.2010.06.003 .

RAJAGURU, R.; MATANDA, M.J. Effects of inter-organizational compatibility on supplychain capabilities: Exploring the mediate role of inter-organizational information systems (IOIS) integration. Industrial Marketing Management 42, p. 620-632, 2013. DOI: http://dx.doi.org/10.1016/j.indmarman.2012.09.002 .

Revista Produção Online, Florianópolis, SC, v.15, n. 3, p. 980-998, jul./set. 2015. 
RIBBKIN, D.; GRIMM, C.M. The impact of cultural differences on buyer-supplier negotiations: An experimental study. Journal of Operations Management 32, p. 114-126, 2014. DOI: http://dx.doi.org/10.1016/j.jom.2014.01.004 .

RIOPEL, D.; CHOUINARD, M.; MARCOTTE, S.; ALY-KADI, D. Ingéneierie et gestion de la logistique inverse: vers des réseaux durables. Paris: Lavoisier, 285p., 2011.

ROOS, G.; ROOS, J. Measuring your company's intellectual performance. Long Range Planning, V. 30, N. 3, pp. 413-426, 1997. DOI: http://dx.doi.org/10.1016/S00246301(97)90260-0.

SANTOS, A.R. Metodologia científica: a construção do conhecimento. 5. ed. Rio de Janeiro: DP\&A, 2002.

STEWART, T.A. Capital intelectual: a nova vantagem competitiva das empresas. Rio de Janeiro: Campus, 1998.

SVEIBY, K.E. A Nova riqueza das organizações: gerenciando e avaliando patrimônios do conhecimento. Rio de Janeiro: Campus, 1998.

SYDLER, R.; HAEFLIGER, S.; PRUSKA, R. Measuring intellectual capital with financial figures: can we predict firm profitability? European Management Journal 32, p. 244-259, 2014. DOI: http://dx.doi.org/10.1016/j.emj.2013.01.008 .

VACHON, S.; KLASSEN, R.D. Green project partnership in the supply chain: the case of package printing industry. Journal of Cleaner Production 14, p. 661-671, 2006. DOI: http://dx.doi.org/10.1016/j.jclepro.2005.07.014 .

VACHON, S.; KLASSEN, R.D. Environmental Management and manufacturing performance: The role of collaboration in supply chain. International Journal Production Economics 111, p. 299-315, 2008. DOI: http://dx.doi.org/10.1016/j.ijpe.2006.11.030 .

VIEGAS, C.V., VARVAKIS, G. J., SELIG, P.M. Strategic Management Accounting (SMA) and Green Supply Chain Management (GSCM): Advancements and Challenges for Integration in Recent Reesearch. In: Varney, Alexandra L. (Org.). Process Control - Theory,

Applications and Challenges - Engineering Tools, Techniques and Tables. 1ed. New York: Nova Science Publishers, V. 1, p. 1-25, 2014.

ZHU, Q.; SARKIS, J.; LAI, K. Institutional-based antecedents and performance outcomes of internal and external green supply chain management practices. Journal of Purchasing \& Supply Management 19, p. 106-117, 2013. http://dx.doi.org/10.1016/j.pursup.2012.12.001.

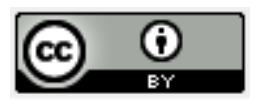

Artigo recebido em 07/12/2014 e aceito para publicação em 11/12/2014

DOI: http://dx.doi.org/ 10.14488/1676-1901.v15i3.1945 